\title{
Mining complex patterns
}

\author{
Annalisa Appice • Michelangelo Ceci · \\ Corrado Loglisci · Elio Masciari • Giuseppe Manco
}

Received: 2 December 2013 / Accepted: 5 December 2013 /

Published online: 8 January 2014

(C) European Union 2014

The recent developments in technologies and life sciences have paved the way to complex interactions among entities in distributed and heterogeneous environments. As a result, an enormous amount of valuable information is available, spanning from structured to multimedia and spatial or spatio-temporal data. The data mining research community has been focusing on developing specialized techniques and algorithms for the analysis of such data. Some of the problems that have received attention include the analysis of multi-table data, XML data, web data, time series and sequences, graphs and trees.

The first International Workshop on New Frontiers in Mining Complex Patterns (NFMCP 2011), held in Bristol UK, on September 24th 2012 in conjunction with the European Conference on Machine Learning and Principles and Practice of Knowledge Discovery in Databases (ECML-PKDD 2012), was aimed at bringing together researchers and practitioners of data mining who are interested in the advances and latest developments in area of extracting patterns from complex data.

\footnotetext{
A. Appice $(\varangle) \cdot$ M. Ceci · C. Loglisci

Dipartimento di Informatica, Università degli Studi di Bari "Aldo Moro" via Orabona, 4-70126 Bari, Italy

e-mail: appice@di.uniba.it

M. Ceci

e-mail: michelangelo.ceci@uniba.it

C. Loglisci

e-mail: corrado.loglisci@uniba.it

E. Masciari · G. Manco

ICAR-Consiglio Nazionale delle Ricerche Rende, Italy

E. Masciari

e-mail: masciari@icar.cnr.it

G. Manco

e-mail: manco@icar.cnr.it
} 
This special issue of the Journal of Intelligent Information Systems (JIIS) features a collection of six papers, representing revised and significantly extended journal versions of the best papers among the eighteen research papers accepted by the program committee of the workshop. These papers went through a rigorous review process to ensure the high quality and comply with JIIS publication standards.

The first paper, "Link Classification with Probabilistic Graphs", by Nicola Di Mauro, Claudio Taranto and Floriana Esposito, proposes two machine learning techniques for the link classification problem in relational data exploiting the probabilistic graph representation. They exploit a language-constrained reachability method to infer the probability of possible hidden relationships that may exists between two nodes in a probabilistic graph.

The second paper, "Hierarchical Object-Driven Action Rules", by Ayman Hajja, Zbigniew W. Ras, and Alicja A. Wieczorkowska, proposes a hybrid action rule extraction approach that combines key elements from both the classical action rule mining approach, and the object-driven action rule extraction approach to discover action rules from object-driven information systems.

The third paper, "Semantic Subgroup Explanations", by Anže Vavpetič, Vid Podpečan and Nada Lavrač, focuses on subgroub discovery (SD), and presents a new approach to explaining subgroups through ontologies and demonstrate its utility on a motivational and on a gene expression profiling use cases.

The fourth paper, "A Method for Reduction of Examples in Relational Learning", by Ondrej Kuzelka, Andrea Szaboova and Filip Zelezny, focuses on relational learning and studies feature selection methods based on the notion of safe reduction and permissive bias of bounded tree width clauses. The authors show that, under this hypothesis bias, examples of arbitrary tree width can be reduced efficiently.

The fifth paper, "A contribution to the discovery of multidimensional patterns in healthcare trajectories", by Elias Egho, Nicolas Jay, Chedy Raissi, Dino Ienco, Pascal Poncelet, Maguelonne Teisseire and Amedeo Napoli, focuses on sequential pattern mining and proposes a new approach to extract heterogenous patterns from complex sequential multidimensional databases.

Finally, the paper "Finding the Most Descriptive Substructures in Graphs with Discrete and Numeric Labels", by Michael C. Davis, Weiru Liu and Paul Miller, focuses on graph datasets with numeric attributes, and proposes a method to discover the descriptive substructures normative both in terms of their structure and in terms of their numeric values.

We are fortunate to have six strong papers in this special issue. We are sure the readers will enjoy them. However, organizing a special section is never easy, and is impossible without the cooperation and help of the authors and the reviewers. We want to thank L. Kerschberg and Z.W. Ras, the Editors-in-Chief of the Journal of Intelligent Information Systems, and all of the authors and reviewers for their constructive and prompt support. 